Research in Science Education, 1997, 27(2), 157-173

\title{
Erratum
}

\section{Conceptual Mediation: A New Perspective on Conceptual Exchange}

Chris Dawson and Harry Lyndon

University of Adelaide

The sentence under Figure 6 should read:

We now have the characteristics of the $\mathrm{A}-\mathrm{B}, \mathrm{A}-\mathrm{Br}$ paradigm, as only the same responses are included in both lists (Figure 6). 